\title{
Ten weeks of aerobic training does not result in persistent changes in VLDL triglyceride turnover or oxidation in healthy men
}

\author{
Birgitte Nellemann, Britt Christensen, Kristian Vissing', Line Thams', \\ Peter Sieljacks ${ }^{1}$, Mads Sørensen Larsen ${ }^{1}$, Jens Otto Lunde Jørgensen and \\ Søren Nielsen
}

Department of Endocrinology and Internal Medicine, Aarhus University Hospital, Nørrebrogade 44, 8000 Aarhus C, Denmark and ${ }^{1}$ Section of Sports Science, Department of Public Health, Aarhus University, Aarhus, Denmark
Correspondence should be addressed to S Nielsen

Email

nielsen.soren@dadlnet.dk

\begin{abstract}
Objective: Very low density lipoprotein triglyceride (VLDL-TG) and free fatty acids (FFA) constitute a substantial proportion of human energy supply both at rest and during exercise. Exercise acutely decreases VLDL-TG concentration, and VLDL-TG clearance is increased after an exercise bout. However, the effects of long-term training are not clear.

Design: The aim was to investigate long-term effects of training by direct assessments of VLDL-TG and palmitate kinetics and oxidation in healthy lean men $(n=9)$ at rest, before and after a 10-week training program, compared with a non-training control group $(n=9)$.

Methods: VLDL-TG kinetics were assessed by a primed constant infusion of $\left[1-{ }^{14} \mathrm{C}\right] \mathrm{VLDL}-\mathrm{TG}$, and VLDL-TG oxidation by specific activity $\left({ }^{14} \mathrm{CO}_{2}\right)$ in expired air. The metabolic study days were placed $60-72 \mathrm{~h}$ after the last exercise bout.

Results: Palmitate kinetics and oxidation were assessed by a $2 \mathrm{~h}$ constant infusion of $\left[9,10-{ }^{3} \mathrm{H}\right]$ palmitate. In the training group $(n=9)$, maximal oxygen uptake increased significantly by $\approx 20 \%(P<0.05)$, and the insulin sensitivity (assessed by the hyperinsulinemic-euglycemic clamp) improved significantly $(P<0.05)$. Despite these metabolic improvements, no changes were observed in VLDL-TG secretion, clearance, or oxidation or in palmitate kinetics.
\end{abstract}

Conclusion: We conclude that 10 weeks of exercise training did not induce changes in VLDL-TG and palmitate kinetics in healthy lean men.

\section{Introduction}

Endurance exercise increases the contribution of fatty acids (FA) for oxidation and reduces the risk of lipidrelated disorders $(1,2,3)$. Postabsorptively, the sources of FA comprise plasma free FA (FFA), originating from adipose tissue lipolysis, intramyocellular triglycerides (TG), and hydrolysis of plasma very low density lipoprotein TG (VLDL-TG). However, the mechanisms involved in regulation of the kinetics of TG-rich lipoproteins, including the quantitative contribution of TG-associated FA for oxidation at rest and in association with exercise, have not been studied in great detail. (c) 2014 European Society of Endocrinology Printed in Great Britain
At present, most studies on the impact of exercise on VLDL-TG kinetics have investigated the effects of acute exercise, either during or shortly after an exercise bout. In untrained individuals, a single bout of exercise reduces fasting VLDL-TG concentration the following day relative to both the intensity and the amount of exercise $(4,5$, $6,7)$, primarily by increasing VLDL-TG clearance $(8,9,10$, $11,12)$. In addition, these acute effects of exercise may be transient, because VLDL-TG clearance tends to normalize as early as $3 \mathrm{~h}$ after exercise (10). On the other hand, more persistent effects of long-term training on VLDL-TG

Published by Bioscientifica Ltd. 
metabolism are plausible. Animal studies indicate that long-term endurance training is associated with decreased hepatic VLDL-TG secretion $(13,14,15)$. Human studies, however, show somewhat divergent results. Long-term training has shown both decreased and unchanged VLDLTG levels $(16,17)$, but whether the reduction (16) was due to reduced hepatic secretion or increased peripheral clearance of VLDL-TG is not clear. On the other hand, 6 months of supervised endurance training was associated with decreased apoB-100 secretion, a measure of VLDL particle number, examined by $\left[1-{ }^{13} \mathrm{C}\right]$ leucine tracer (18). This method, however, does not provide information regarding either TG content of the VLDL particles or VLDL-TG FA oxidation. Other kinetic studies using glycerol precursor labeling of VLDL-TG in combination with mathematical modeling have found both decreased (19) and unaltered (20) hepatic VLDL-TG secretion rates following training programs. The differences between these and the present studies may be due to differences in the intensity and duration of the training programs as well as in the volunteers studied. Importantly, however, the methods used do not allow direct measurements of VLDL-TG FA oxidation, which is central to understanding the mechanisms underlying prolonged effects of training on systemic VLDL-TG metabolism.

Previous studies have reported $(21,22,23)$ that postabsorptive VLDL-TG FA accounts for $10-15 \%$ of whole-body resting energy expenditure (REE) in healthy sedentary humans $(22,23,24)$. Moreover, VLDL-TG secretion and clearance rates were significantly decreased during acute exercise as well as during the immediate recovery of the exercise bout of $90 \mathrm{~min}$ at $50 \%$ of maximal oxygen uptake $\left(\mathrm{VO}_{2} \mathrm{max}\right)$, whereas VLDL-TG FA oxidation rate was largely unchanged compared with resting levels (22).

Exercise also improves insulin sensitivity directly for about $48 \mathrm{~h}$ after an exercise bout $(25,26)$, and insulin modulates hepatic VLDL-TG secretion. For example, insulin acutely suppresses hepatic VLDL-TG secretion, while insulin resistance is associated with increased hepatic VLDL-TG secretion rates both in the basal state and during insulin stimulation $(27,28)$. Therefore, we hypothesized that a persistent improvement in insulin sensitivity in response to a prolonged training intervention would also lead to sustained changes in VLDL-TG and palmitate kinetics. In this study, we investigated the impact of a 10-week training intervention in healthy, sedentary men on plasma VLDL-TG and FFA kinetics and oxidation using i.v. infusion of $\left[1-{ }^{14} \mathrm{C}\right]$ VLDL-TG and $\left[9,10-{ }^{3} \mathrm{H}\right]$ palmitate in combination with measurements of plasma isotopic specific activity (SA) and exhaled ${ }^{14} \mathrm{CO}_{2}$ before and after the 10-week intervention period.

\section{Subjects and methods}

\section{Subjects}

Eighteen healthy young men were studied as part of a larger study involving training ( $\mathrm{Tr}$ ) intervention, erythropoiesis-stimulating agent (Darbepoetin alfa) treatment as compared with a physically inactive control (C) group. This study examined VLDL-TG and FFA (palmitate) kinetics and oxidation rates of the participants in the $\mathrm{C}$ and $\mathrm{Tr}$ subgroups with no injections of erythropoiesisstimulating agent. Inclusion criteria (29) required that all participants were untrained, with a $\mathrm{VO}_{2} \max <50 \mathrm{ml} / \mathrm{kg}$ per min. Additionally, all were normotensive, nonsmokers, used no medication, and had a normal blood count, lipid profile, fasting plasma glucose, $\mathrm{HbA1c}$, and a normal electrocardiogram. The study was registered on www.clinicaltrials.gov (NCT01320449). The Regional Scientific Ethics Committee of Central Denmark approved the study protocol (M-20110035), and written informed consent was obtained from all participants.

\section{Experimental design}

A detailed description of the study design has been reported elsewhere (29). In brief, in a randomized, paired design, subjects were allocated to a $\mathrm{C}$ group $(n=9)$ or a $\mathrm{Tr}$ group $(n=10)$. One participant in the $\operatorname{Tr}$ group was excluded from this study compared with the overall study due to incomplete data. Additionally, for two further participants in the Tr group, it was not possible to measure palmitate oxidation. $\mathrm{VO}_{2} \mathrm{max}$ was determined by an incremental cycling protocol (starting at 140 watt and 35 watt increase/min) until exhaustion on a stationary exercise bike (Monark, Ergomedic 828E, Varberg, Sweden) before inclusion and after the last study day in both groups. The $\operatorname{Tr}$ group participated in supervised endurance bicycle exercise thrice weekly for 10 weeks (30). The training protocol consisted of three different weekly exercise sessions. After a 5-min warm-up, one of three training sessions was performed: 'Session 1', $40 \mathrm{~min}$; 'Session 2', $2 \times 20 \mathrm{~min}$; or 'Session 3 ', $8 \times 5 \mathrm{~min}$, thus comprising both high-intensity aerobic exercise and moderate-intensity endurance exercise. Sessions were performed at an average of $65 \%$ of watt-max. Lipid kinetic studies were carried out under resting conditions at baseline and after 10 weeks. In order to avoid any acute 
effects of exercise, the post-training metabolic study day was scheduled 60-72 h after the last exercise bout. Moreover, the participants were instructed to abstain from other strenuous exercise and alcohol consumption 2 days before the metabolic study days, and not to change their diet for 3 days before each metabolic study day.

\section{Metabolic study day}

Participants fasted from $1000 \mathrm{~h}$ the evening before the study days (only tap or mineral water was allowed). In the morning, volunteers arrived by taxi, and were immediately admitted to the Research Unit and instructed to avoid unnecessary physical activity. The subjects were studied in the recumbent position, wearing light hospital clothing at an ambient room temperature of $20-22^{\circ} \mathrm{C}$. Two catheters were placed intravenously, one in the antecubital vein for infusions and one by a heated-hand vein technique in order to obtain arterialized blood. Each study day consisted of a 4 -h basal period ( $t=0-240 \mathrm{~min}$ ) with primed continuous autologous $\left[1-{ }^{14} \mathrm{C}\right] \mathrm{VLDL}-\mathrm{TG}$ infusion followed by a 2-h hyperinsulinemic-euglycemic clamp performed for the assessment of insulin sensitivity. The insulin (Actrapid, Novo Nordisk, Hellerup, Denmark) infusion rate was set at $0.6 \mathrm{mU} / \mathrm{kg}$ per min and blood glucose was measured every $10 \mathrm{~min}$ and clamped at $5.0 \mathrm{mmol} / \mathrm{l}$ by a variable $20 \%$ glucose infusion. The glucose infusion rate during the final $30 \mathrm{~min}$ was considered steady state and used as a measure of insulin sensitivity ( $M$-value). Moreover, a constant 2-h infusion of $\left[9,10-{ }^{3} \mathrm{H}\right]$ palmitate was performed from $T=120-240 \mathrm{~min}$ for the measurement of palmitate turnover and oxidation rates. Steady state blood samples and breath samples were collected during the last $30 \mathrm{~min}$ of the basal period ( $T=$ 210,225 , and $240 \mathrm{~min}$ ) for the measurement of concentration and SA of VLDL-TG and palmitate in plasma and ${ }^{14} \mathrm{CO}_{2}$ in breath. Indirect calorimetry was performed from $T=60-90 \mathrm{~min}$. After the clamp, all catheters were removed, the participants had lunch and when blood glucose had stabilized they were dismissed.

\section{VLDL-TG tracer preparation}

One week before the metabolic study day, the participants attended the Research Unit after a $12 \mathrm{~h}$ overnight fast. An $80 \mathrm{ml}$ blood sample was obtained aseptically to isolate VLDL-TG for ex vivo labeling as previously described (21) with minor modifications. Plasma was separated and then sonicated with $15 \mu \mathrm{Ci}$ of $\left[1-{ }^{14} \mathrm{C}\right]$ triolein (PerkinElmer, Waltham, MA, USA) at $5{ }^{\circ} \mathrm{C}$ for $2 \mathrm{~h}$.
The $\left[1-{ }^{14} \mathrm{C}\right]$ triolein-labeled plasma was transferred to sterile tubes and covered with a saline solution of $d=1.006 \mathrm{~g} / \mathrm{cm}^{3}$ and ultracentrifuged $(50.3 \mathrm{Ti}$ rotor $(37000 \boldsymbol{g})$ or $50.4 \mathrm{Ti}$ rotor $(37000 \boldsymbol{g})$, Beckman Instruments, Inc. (Palo Alto, CA, USA)) for $18 \mathrm{~h}$ and at $10^{\circ} \mathrm{C}$. The labeled VLDL-TG fraction in the supernatant was obtained with a modified sterile Pasteur pipette, and the solution passed through a Millipore filter with a pore size of $0.20 \mu \mathrm{m}$. The VLDL-TG tracer was stored under sterile conditions at $5{ }^{\circ} \mathrm{C}$ and a representative sample of the labeled $\left[1-{ }^{14} \mathrm{C}\right]$ VLDL-TG from all participants was cultured to ensure sterility before autologous infusion.

\section{Plasma VLDL-TG concentration and SA}

VLDL-TG was separated from $\sim 3 \mathrm{ml}$ plasma by ultracentrifugation as described above. The VLDL fraction in the top layer was obtained by slicing the tube $\approx 1 \mathrm{~cm}$ from the top with a tube slicer (Beckman Instruments, Inc.) and the exact volume was noted. A 300- $\mu$ l aliquot of the solution was analyzed (Glycerol blanked assay; COBAS c111, Roche) for TG content and the plasma concentration of VLDL-TG was calculated. Scintillation fluid was added to the remaining solution and $\left[{ }^{3} \mathrm{H}\right]$ and $\left[{ }^{14} \mathrm{C}\right] \mathrm{SA}$ was measured by dual channel liquid scintillation counting to $<2 \%$ counting error.

\section{Breath ${ }^{14} \mathrm{CO}_{2}$-specific activity}

[1- ${ }^{14}$ C]VLDL-TG FA oxidation was calculated from the activity of ${ }^{14} \mathrm{CO}_{2}$ in expired air. Breath samples were collected in breath bags (IRIS-breath-bags; Wagner Analysen Technik, Bremen, Germany) and the air was passed through a solution containing $0.5 \mathrm{ml}$ hyamine hydroxide in $1 \mathrm{M}$ methanol, $2 \mathrm{ml} \mathrm{96 \%} \mathrm{ethanol,} \mathrm{and} \mathrm{one} \mathrm{to} \mathrm{two} \mathrm{drops}$ of phenolphthalein. A color change occurred when $0.5 \mathrm{mmol} \mathrm{CO}_{2}$ was trapped in the solution, and $\left[{ }^{14} \mathrm{C}\right]$ activity was measured by liquid scintillation counting to $<2 \%$ counting error.

\section{Palmitate turnover and oxidation}

Systemic palmitate turnover and oxidation were measured using the isotope dilution technique with a 2-h constant infusion from $T=120-240$ min of $\left[9,10-{ }^{3} \mathrm{H}\right]$ palmitate $(0.3 \mu \mathrm{Ci} / \mathrm{min}$; Department of Clinical Physiology and Nuclear Medicine, Aarhus University Hospital, Denmark) (31). Plasma palmitate concentration and SA were measured at baseline and at $T=150,160,170,180,195$, 210,225 , and $240 \mathrm{~min}$ by HPLC using $\left[{ }^{2} \mathrm{H}_{31}\right]$ palmitate as 
an internal standard. Steady state SA was verified for each individual at $T=150,160,170$, and $180 \mathrm{~min}$. Palmitate flux $(\mu \mathrm{mol} / \mathrm{min})$ was calculated as $\left[9,10-{ }^{3} \mathrm{H}\right]$ palmitate infusion rate (d.p.m./min) divided by the steady-state palmitate SA (d.p.m./ $\mu \mathrm{mol}$ ). Oxidation of plasma palmitate was measured using the slope of the increase in ${ }^{3} \mathrm{H}_{2} \mathrm{O}$ in total body water vs time to calculate ${ }^{3} \mathrm{H}_{2} \mathrm{O}$ production rate $(T=120-240 \mathrm{~min})$. The plasma $\left[{ }^{3} \mathrm{H}_{2} \mathrm{O}\right]$ concentration was determined as described previously (32).

\section{Body composition}

Total body fat mass (FM), leg fat, fat percentage, and fat free mass (FFM) were measured by the dual-energy X-ray absorptiometry (QDR-2000, Hologic, Marlborough, MA, USA) at the end of each metabolic study days.

\section{Indirect calorimetry}

REE and respiratory exchange ratio (RER) were measured by indirect calorimetry (Deltatrac monitor, Datex Instrumentarium, Helsinki, Finland). Net lipid and glucose oxidation were calculated after correction for protein oxidation (33). Protein oxidation was calculated from urea excretion in urine collected during the basal period.

\section{Calculations}

VLDL - TG secretion rate ( $\mu \mathrm{mol} / \mathrm{min})$ :

VLDL - TG infusion rate/steady state VLDL - TG SA

VLDL - TG clearance ( $\mathrm{ml} / \mathrm{min})$ :

VLDL - TG secretion rate/( $\mathrm{C}_{\mathrm{VLDL}}$ - TG $)$
VLDL-TG oxidation

Fractional VLDL - TG oxidation (\%) of the infused tracer :

$$
\left({ }^{14} \mathrm{CO}_{2} \mathrm{SA} \times \mathrm{VCO}_{2}\right) /(k \times \mathrm{Ar} \times F),
$$

where $k$ is the volume of $\mathrm{CO}_{2}$ at $20{ }^{\circ} \mathrm{C}$ and one atomic pressure $(22.4 \mathrm{l} / \mathrm{mol}), \mathrm{Ar}$ is the fractional acetate carbon recovery factor in breath $\mathrm{CO}_{2}$ (0.56 at rest) (34), and $F$ is the tracer infusion rate.

Total VLDL - TG oxidation rate $(\mu \mathrm{mol} / \mathrm{min})$ :

Fractional VLDL - TG oxidation

$\times$ VLDL - TG secretion rate.

VLDL-TG FA oxidation rate $(\mu \mathrm{mol} / \mathrm{min})$ was calculated by multiplying VLDL-TG oxidation rate by 3 (three FA per TG molecule). To calculate energy production (kcal/day) from VLDL-TG FA, the rate of VLDL-TG FA oxidation was converted to its weight equivalent using the molecular weight of $282 \mathrm{~g} / \mathrm{mol}$ of oleic acid and multiplied by the caloric density of $9.1 \mathrm{kcal} / \mathrm{g}$ and $1440 \mathrm{~min} /$ day.

Plasma FFA oxidation (kcal/24 h):

(palmitate ox $(\mu \mathrm{mol} / \mathrm{min}) \times 1440 \mathrm{~min} \times 256.42 \mathrm{~g} / \mathrm{mol}$ $\times 9.1 \mathrm{kcal} / \mathrm{g}) /(0.29 \times 0.8 \times 1000000)$,

where 0.29 is the average ratio of palmitate in TG long chain $\mathrm{FA}$, and 0.8 is the recovery factor for ${ }^{3} \mathrm{H}$ in ${ }^{3} \mathrm{H}_{2} \mathrm{O}(35)$, and $256.42 \mathrm{~g} / \mathrm{mol}$ is the molecular weight of palmitate. The relative contribution of FFA and VLDL-TG FA to oxidation was calculated as the percentage of the total lipid oxidation.

Table 1 Subject characteristics and anthropometry.

Age
Weight (kg)
BMI
Waist (cm)
FFM (kg)
Fat mass (kg)
Leg fat (kg)
Fat percentage (\%)
Total cholesterol (mM)
HDL-cholesterol (mM)
LDL-cholesterol (mM)
Hemoglobin (mM)

\begin{tabular}{c}
\hline C-before \\
\hline $26.1 \pm 1.6$ \\
$79.2 \pm 3.3$ \\
$24.0 \pm 1.0$ \\
$84(78-103)$ \\
$61.0 \pm 2.4$ \\
$18.2 \pm 1.9$ \\
$6.2 \pm 0.5$ \\
$21.3 \pm 1.5$ \\
$3.8 \pm 0.2$ \\
$1.2 \pm 0.1$ \\
$2.2 \pm 0.2$ \\
$9.2 \pm 0.1$ \\
\hline
\end{tabular}

$\begin{array}{r}\hline \text { C-after } \\ 79.1 \pm 3.0 \\ 24.0 \pm 1.0 \\ 59.9 \pm 2.1 \\ 19.2 \pm 1.6 \\ 6.4 \pm 0.5 \\ 21.9 \pm 1.4 \\ 3.7 \pm 0.2 \\ 1.2 \pm 0.1 \\ 2.1 \pm 0.2 \\ 9.1 \pm 0.1 \\ \hline\end{array}$

\begin{tabular}{c}
\hline Tr-before \\
\hline $22.1 \pm 0.7^{\dagger}$ \\
$75.2 \pm 2.8$ \\
$22.6 \pm 0.7$ \\
$81(74-99)$ \\
$61.3 \pm 2.4$ \\
$14.0 \pm 4.0^{\dagger}$ \\
$6.4 \pm 0.5$ \\
$18.4 \pm 1.5$ \\
$4.1 \pm 0.3$ \\
$1.2 \pm 0.1$ \\
$2.5 \pm 0.3$ \\
$9.3 \pm 0.1$ \\
\hline
\end{tabular}

\begin{tabular}{ccc}
\cline { 1 - 1 } Tr-after & & ANOVA \\
$75.2 \pm 2.8$ & & 0.85 \\
$22.5 \pm 0.6$ & & 0.74 \\
& & \\
$61.5 \pm 2.3$ & & 0.25 \\
$13.7 \pm 3.9^{\dagger}$ & & 0.17 \\
$4.8 \pm 0.5^{*}$ & & 0.01 \\
$17.1 \pm 1.4^{*}$ & & 0.01 \\
$4.1 \pm 0.3$ & & 0.67 \\
$1.2 \pm 0.1$ & & 0.84 \\
$2.4 \pm 0.3$ & & 0.22 \\
$9.0 \pm 0.2$ & & 0.08
\end{tabular}

* vs before, $\dagger$ vs control. For age and waist the statistic test was Students $t$-test, for all other parameters RM-NOVA was used. Data shown as mean \pm S.E.M./median (range), * vs before, † vs control. C, $n=9 ; \operatorname{Tr}, n=9 ; \mathrm{C}$, control group; Tr, training group; LBM, lean body mass. 


\section{Statistical analyses}

Data are presented as mean \pm s.E.M. or as median (range). Normality of data was checked by QQ-plots, plots of residuals, and equal variance test. Differences at baseline were examined by Student's t-test or Mann-Whitney's two-sample test for parametric and non-parametric data respectively. Differences between the effects of exercise and non- exercise regarding kinetic parameters, hormones, substrates, and body composition were examined using two-way ANOVA for repeated measurements; if the ANOVA was significant post hoc analysis was performed (Student-Newman-Keul test). Non-parametric data were logarithmic transformed before ANOVA analysis to ensure normal distribution. Within group changes in the abovementioned parameters were analyzed using Student's paired $t$-test or Wilcoxon's signed-rank test for parametric and non-parametric data respectively. Since no previous data of the impact of long-term training on VLDL-TG FA measured directly exist, we performed a power analysis based on data showing insulin suppression of VLDL-TG secretion of $36 \pm 17 \%$ (mean \pm s.D.) in healthy men (28). We considered half of that reduction, i.e. $18 \pm 17 \%$, to be relevant. Thus, with $\beta=0.80$ and $\alpha=0.05$, the sample size was calculated to $n=16$ (two sample tests) and $n=8$ (paired $t$-test). Statistical significance was set at $P<0.05$.

\section{Results}

Subject characteristics and anthropometric data are summarized in Table 1 . Total body weight, BMI, FM, and FFM were similar in the two groups. Exercise was associated with a significant decrease in leg fat and in fat percentage.

During the training intervention compliance was $97.0 \pm 0.9 \%$, and the participants spent on average $622 \pm$ $27 \mathrm{kcal} /$ exercise bout. $\mathrm{VO}_{2} \max$ was not different between the groups at baseline. However, 10 weeks of training improved the maximal oxygen uptake by $\approx 20 \%$ in the $\mathrm{Tr}$ group compared with the baseline level $\left(\mathrm{VO}_{2} \mathrm{max}\right.$ (ml/kg per min) C-before: 44.4 (26.0-44.9); C-after: 46.4 (24.6-48.8); Tr-before: 43.5 (35.9-49.6); Tr-after: 51.8 (47.8-56.4), ANOVA, $P<0.001)$. In addition, insulin sensitivity improved in the $\mathrm{Tr}$ group compared with $\mathrm{C}$ ( $M$-value (mg/kg per min) C-before: $3.5 \pm 0.5$; C-after: $3.8 \pm 0.9$, Tr-before: $3.5 \pm 0.5$; Tr-after: $5.0 \pm 0.5$, ANOVA, $P=0.02$ ). Conversely, REE (REE (kcal/24 h) C-before: $1705 \pm 48$; C-after: $1696 \pm 40 ;$ Tr-before: $1716 \pm 54$; Tr-after: $1817 \pm 69$, ANOVA, $P=0.32$ ) and RER (RER: C-before: $0.84 \pm 0.01$; C-after: $0.84 \pm 0.01$; Tr-before:
$0.82 \pm 0.01$; Tr-after: $0.83 \pm 0.01$, ANOVA, $P=0.39$ ) were not significantly different between the groups and were not affected significantly by the intervention.

\section{Substrate concentrations}

Basal plasma TG, VLDL-TG, FFA, and insulin concentrations were not significantly different between the groups at baseline, and the concentrations were not
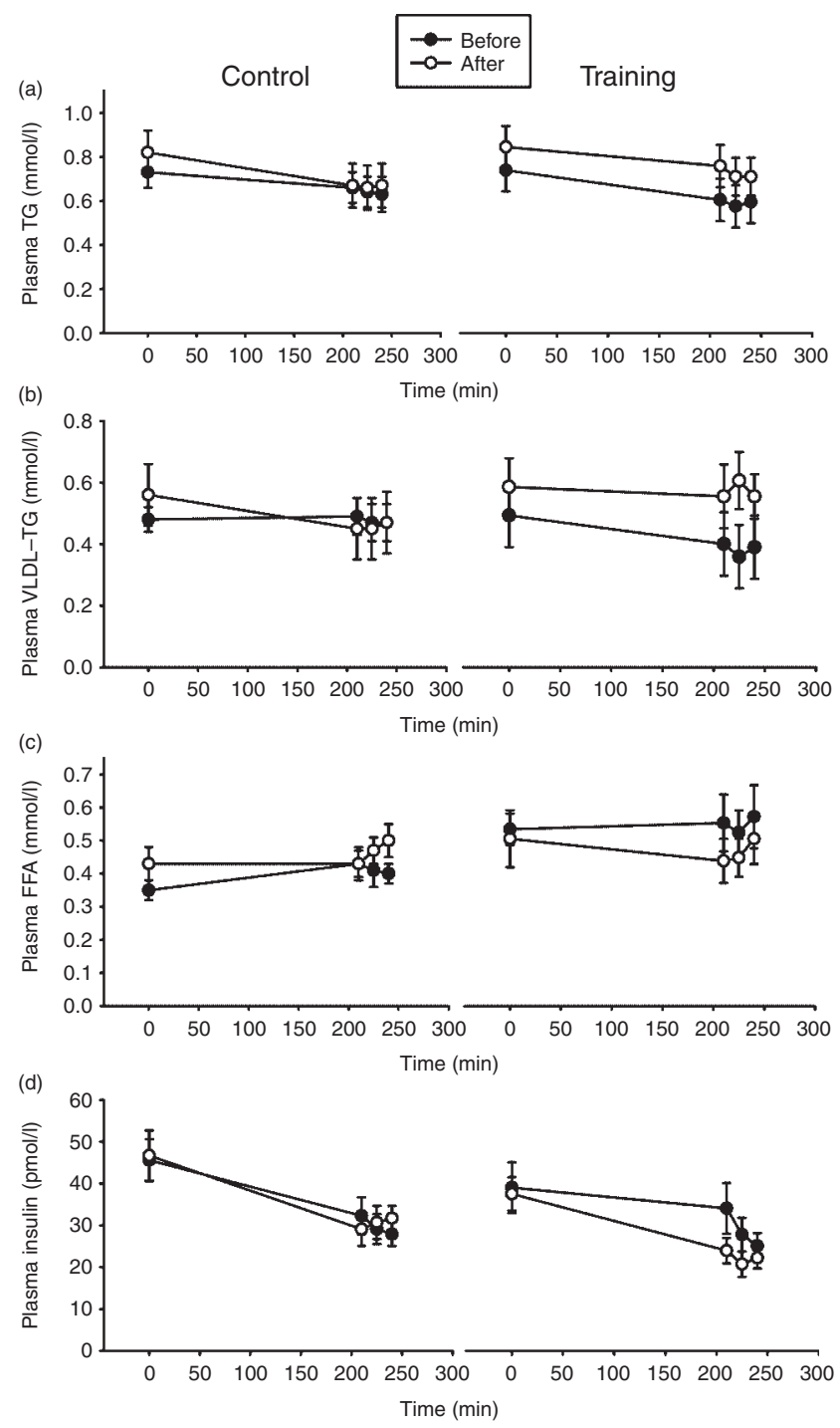

Figure 1

Concentrations of (a) plasma TG, (b) plasma VLDL-TG, (c) plasma FFA, and (d) plasma insulin before (black circles) and after (white circles) 10 weeks' intervention for control (left side) and training (right side) groups respectively. Mean and S.E.M. error bars. C, $n=9 ; \operatorname{Tr}, n=9$; statistic test, ANOVA. 

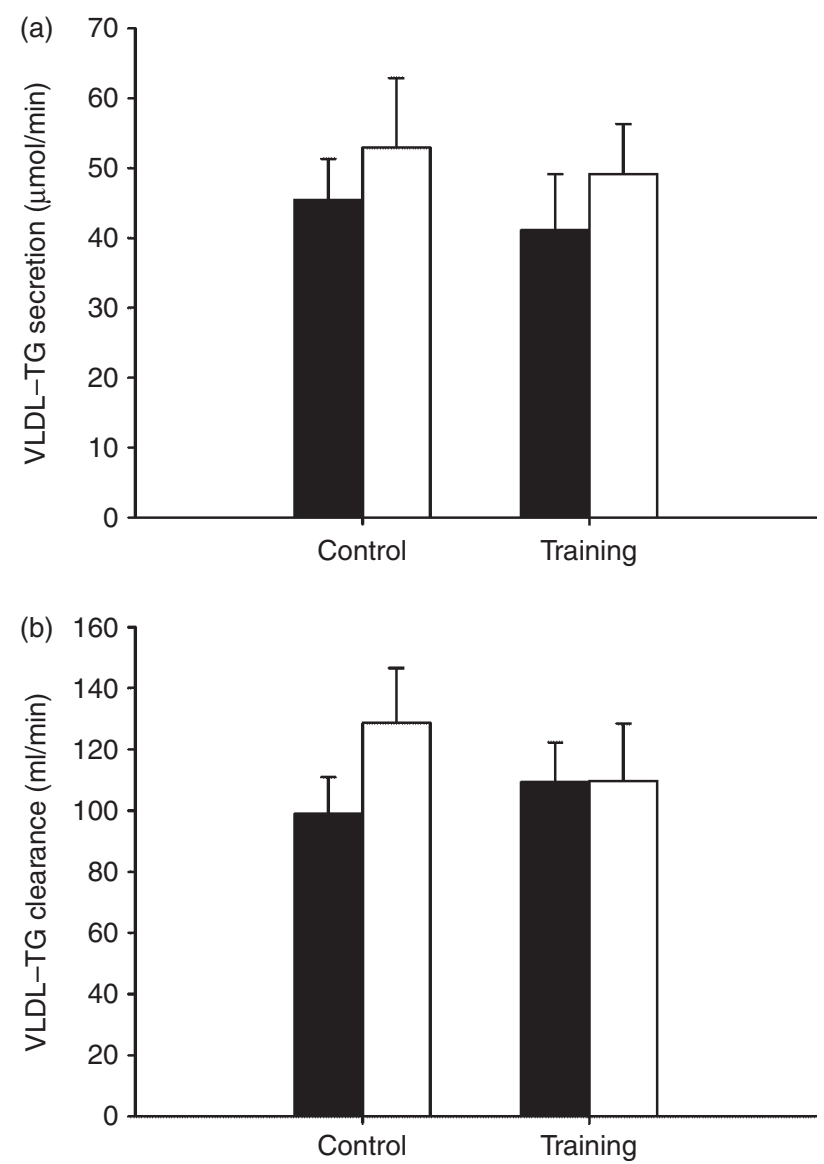

Figure 2

VLDL-TG secretion rate (a) and clearance rate (b) before and after 10 weeks' intervention for $\mathrm{C}$ and $\mathrm{Tr}$ groups respectively. Black bar before, white bar after, mean and S.E.M. error bars. C, $n=9$; $\operatorname{Tr}, n=9$; statistic test, ANOVA.

altered significantly during the intervention period in either group (Fig. 1).

\section{VLDL-TG kinetics and oxidation}

VLDL-TG secretion and clearance rates are depicted in Fig. 2. No significant differences in VLDL-TG secretion or clearance rates were present at baseline. Moreover, no significant change was noted during the intervention, and no significant difference between the groups occurred during the intervention.

Fractional VLDL-TG oxidation was similar $\approx 50 \%$ (Fig. 3a) in both groups and did not differ significantly between groups during the intervention period (ANOVA, $P=0.90)$. Moreover, total VLDL-TG FA oxidation rate (Fig. 3b) was not significantly different between the groups at baseline and remained stable during the intervention. No significant difference was observed between the groups during the intervention (ANOVA, $P=0.17$ ).

\section{Palmitate turnover and oxidation}

Palmitate turnover was not significantly different between the groups at baseline and remained unchanged during the intervention. No significant difference was found between the groups during the intervention (Fig. 4a). A slight, though not significant decrease was noted in the Tr group (post hoc $P=0.06$ ) but not in the $\mathrm{C}$ group (post hoc $P=0.19)$. Absolute palmitate oxidation rates were not significantly different between the groups at baseline, and the change in palmitate oxidation was not significantly
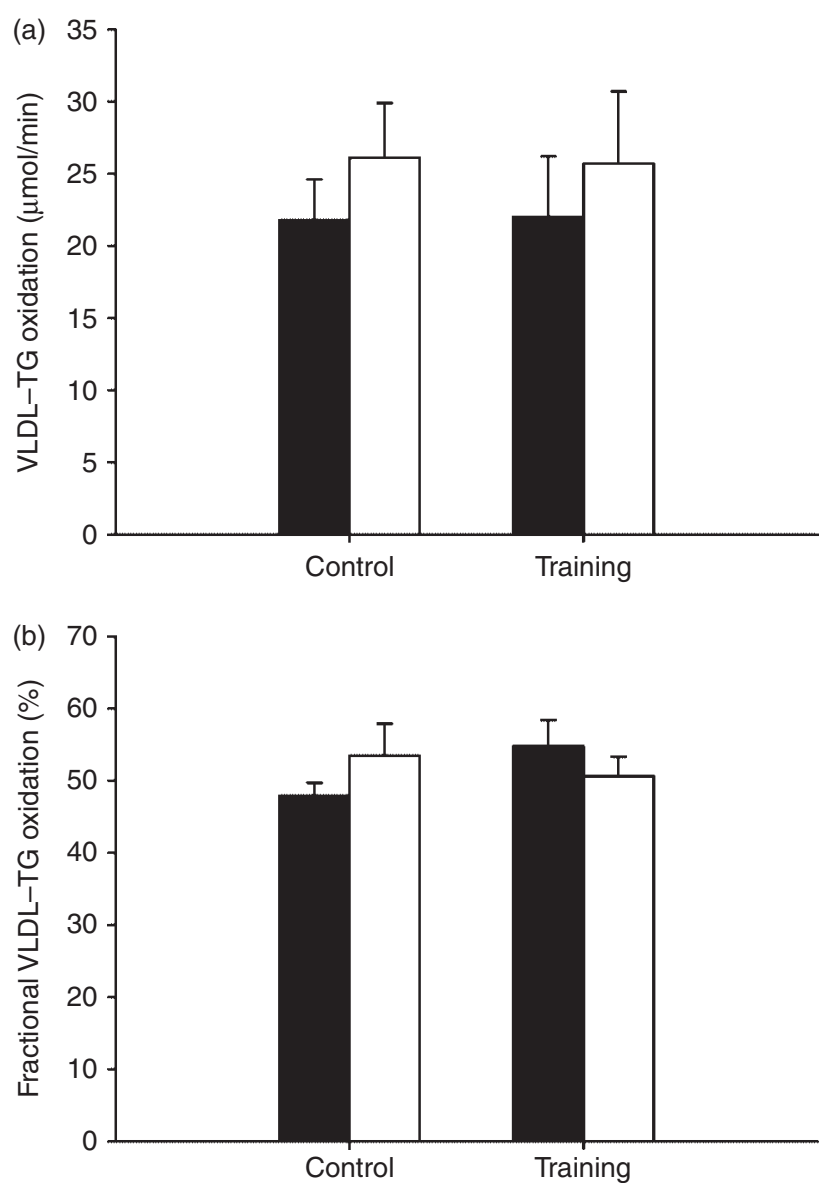

\section{Figure 3}

VLDL-TG oxidation (a) and fractional oxidation (b) before and after 10 weeks' intervention for $C$ and $\operatorname{Tr}$ groups respectively. Black bar before, white bar after, mean and S.E.M. error bars. $Y$-axis in (b) is logarithmic transformed. $C, n=9 ; \operatorname{Tr}, n=9$; statistic test, ANOVA. 

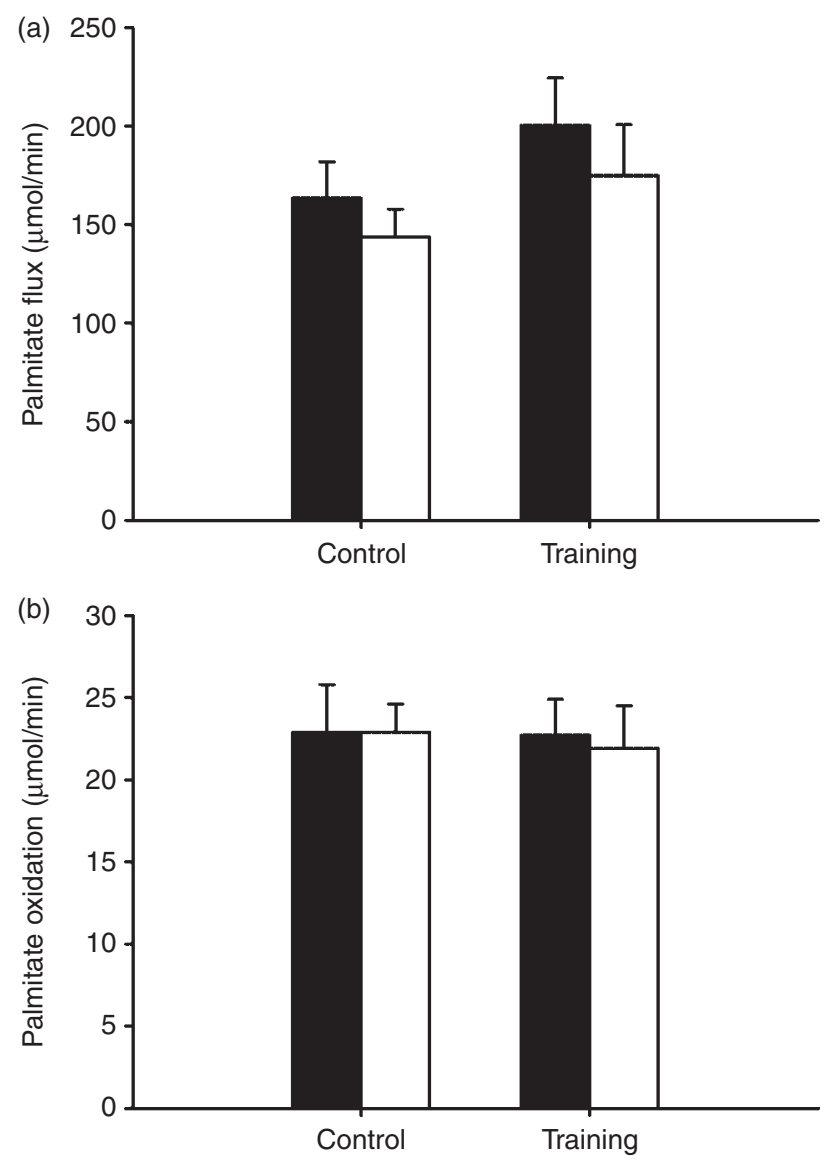

Figure 4

Palmitate turnover (a) and absolute palmitate oxidation

(b) before and after 10 weeks' intervention for $\mathrm{C}$ and $\mathrm{Tr}$ groups respectively. Black bar before, white bar after, error bars:

S.E.M. C, $n=9 ; \operatorname{Tr}, n=9$ palmitate turnover and $n=7$ palmitate oxidation; statistic test, ANOVA.

different between the groups (Fig. 4b). The fractional palmitate oxidation was not significantly different between the groups at baseline or after the intervention period; however, a small but significant increase was observed in the $\mathrm{C}$ group (fractional palmitate oxidation (\% of infused tracer) C-before: $13.80 \pm 0.01$; C-after: $15.90 \pm 0.01$; Tr-before: $13.00 \pm 0.01$; Tr-after: $14.10 \pm$ 0.01, ANOVA, $P=0.02$ ).

\section{Glucose oxidation}

Glucose oxidation was not significantly different between the groups and was not altered during the intervention (glucose oxidation (kcal/24 h) C-before: 29.8 \pm 3.4 ; C-after 23.6 \pm 2.4 ; Tr-before: $24.0 \pm 4.5$; Tr-after: 30.9 \pm 2.0 , ANOVA, $P=0.68$ ).

\section{Lipid oxidation}

There were no significant difference in total lipid oxidation at baseline, nor were there any differences in the relative distribution of plasma FFA oxidation and VLDL-TG FA oxidation, whether this was expressed as total amounts or as percentage of the total lipid oxidation (Table 2). Also, the intervention did not result in any significant changes in the contribution of the different lipid sources for oxidation in either group. The equivalent energy production attributed to VLDL-TG FA oxidation (Table 2) resulted in comparable relative contributions of basal VLDL-TG FA oxidation with REE of $\approx 15 \%$ before and after intervention in both groups (ANOVA, $P=0.32$ ).

\section{Discussion}

In this study, we examined the effect of a long-term training program on VLDL-TG FA and palmitate kinetics, and its relationship with whole-body lipid and glucose metabolism. Despite the expected improvement in $\mathrm{VO}_{2} \mathrm{max}$ and whole-body insulin sensitivity, there was no persistent effect of 10 weeks of training on VLDL-TG FA secretion, clearance, or oxidation rates. Additionally, neither REE, RER, nor palmitate turnover or oxidation

Table 2 Distribution of total lipid oxidation between FFA, VLDL-TG FA, and the residual lipid oxidation. For one participant in Tr it was not possible to measure palmitate oxidation due to lack of material. Data shown as mean \pm s.E.M./median (range).

\begin{tabular}{|c|c|c|c|c|c|}
\hline & C-before & C-after & Tr-before & Tr-after & ANOVA \\
\hline Total lipid ox (kcal/h) & $27(10-37)$ & $24(11-35)$ & $28(24-52)$ & $38(22-59)$ & 0.18 \\
\hline FFA ox (kcal/h) & $13.8 \pm 1.7$ & $13.2 \pm 1.4$ & $13.8 \pm 2.8$ & $13.3 \pm 1.6$ & 0.88 \\
\hline FFA ox (\% of total lipid ox) & $50 \pm 7$ & $57 \pm 9$ & $45 \pm 4$ & $44 \pm 6$ & 0.64 \\
\hline VLDL-TG FA ox (kcal/h) & $3.4 \pm 0.4$ & $4.0 \pm 0.6$ & $3.4 \pm 0.7$ & $4.0 \pm 0.8$ & 0.29 \\
\hline VLDL-TG FA ox (\% of total lipid ox) & $37 \pm 7$ & $41 \pm 8$ & $36 \pm 6$ & $34 \pm 8$ & 0.13 \\
\hline Residual lipid ox (kcal/h) & $2.5 \pm 3.8$ & $-0.8 \pm 4.1$ & $6.9 \pm 2.4$ & $4.9 \pm 4.5$ & 0.25 \\
\hline
\end{tabular}

C, $n=9$; $\operatorname{Tr}, n=7$; Ox, oxidation; C, control group; Tr, training group; FFA, free fatty acids; VLDL-TG, very LDL triglyceride; FA, fatty acids. 
were significantly altered by training as compared with control subjects.

This is the first study to perform combined direct measurements of whole-body VLDL-TG and palmitate kinetics and oxidation before and after long-term supervised endurance training in healthy sedentary men. Most previous studies have examined VLDL-TG kinetics in relation to a single bout of exercise and reported increased VLDL-TG clearance of short duration (1 day) $(9,10$, $12,22)$. In order to investigate the long-term effects of training while at the same time avoiding interference of any residual effects induced by acute exercise, this study was designed so that the metabolic study day was placed 60-72 $\mathrm{h}$ after the last exercise bout. This time frame was chosen because it has been shown in many studies that measurable improvements in both insulin sensitivity to glucose metabolism and in physical performance capacity $\left(\mathrm{VO}_{2} \mathrm{max}\right)$ persists after this time, whereas improvement in TG concentrations do not change. Thus, the question arises whether persistent changes in VLDL-TG kinetics, as opposed to VLDL-TG concentration measurements, are also present at this prolonged time point. Although we found a persistent increase in whole-body insulin sensitivity $60-72 \mathrm{~h}$ after the last exercise bout, we could not demonstrate a concomitant change in basal hepatic VLDL-TG secretion or palmitate turnover. To our knowledge, only one other study has investigated long-term training effects on VLDL-TG kinetics in healthy non-obese men $48 \mathrm{~h}$ after the last exercise bout (19), using infusion of a glycerol precursor followed by mathematical modeling of the plasma tracer data. The authors performed an endurance training protocol for 8 weeks in healthy men, which showed no significant changes in body weight or plasma levels of FFA and TG. Interestingly, however, $48 \mathrm{~h}$ after the last exercise bout they did observe an increase in VLDL-TG secretion but no difference in clearance rates (19). We speculate that this finding could be a temporary state in the metabolic adaptation (i.e. increased secretion) that may occur after the immediate TG redistribution period in healthy lean men. However, methodological aspects may also have influenced the results. Finally, with this indirect approach it is not possible to calculate substrate oxidation, which is pivotal in all exercise studies (19).

Assessment of VLDL-TG FA oxidation has previously proven to be methodologically challenging. However, in this study we used a novel and validated method that allows direct measurements of VLDL-TG FA oxidation (21). VLDL-TG contributes significantly to energy expenditure (EE) at rest $(10-15 \%)(23,24)$, but the effective amount oxidized remains unaltered during acute aerobic exercise in healthy lean subjects (22). In this study, we extend these findings to include an unchanged VLDL-TG FA oxidation following long-term training in healthy men. Thus, it appears that in healthy adults any effects on VLDL-TG metabolism are temporary and disappear within a few hours after an exercise bout. It cannot be excluded, however, that other training modalities such as resistance exercise, more prolonged training periods, or exercise of higher intensity, could have yielded a different outcome. Still, the training program used did improve both physical performance and insulin sensitivity. Interestingly, some studies have shown that the combination of endurance and resistance exercise is more potent in lowering the concentrations of TG, LDL-cholesterol, and apoB-100 compared with either exercise form separately $(36,37)$. This could indicate that resistance exercise might possess a different potential in the modulation of VLDL-TG kinetics. In this study, we find that resting lipid oxidation originating from other sources than plasma FFA and VLDL-TG FA, i.e. i.m. TG, are negligible (Table 2), which is consistent with studies done in obese individuals at rest (38) and during very-low intensity exercise (39).

Only few studies have been able to modulate postabsorptive VLDL-TG oxidation. Consumption of a high-fat diet showed increased leg VLDL-TG net clearance during exercise compared with a high-carbohydrate diet $(40,41)$; this finding was unaffected by VLDL-TG concentration and training program (40). However, measurement of leg $a-v$ differences, which was used in these studies, does not allow differentiation between increased storage of VLDL-TG in leg fat vs muscle tissue or to calculate oxidation, which therefore could explain some of the observed difference in leg clearance. Conversely, in a previous study, we reported that growth hormone infusion did not change VLDL oxidation in healthy adults although FFA levels and whole-body lipid oxidation were increased $(42,43)$.

The previously mentioned acute kinetic changes during exercise and in the early recovery period (22) could indicate that a redistribution of TG and VLDL-TG takes place during that period, replenishing TG depots in, for example, muscle and liver during the recovery period, thereby potentially improving the ability to meet energy needs. The data from this study lends indirect support to the theory of a temporary acute TG FA redistribution, as the hepatic secretion rate was unchanged $60-72 \mathrm{~h}$ after the last exercise bout, despite improved physical performance and insulin sensitivity. The lack of a persistent effect of endurance training on VLDL-TG kinetics and 
concentration may be explained by the relatively short upregulation (20-30 h post exercise) in lipoprotein lipase (LPL) activity in response to an acute exercise bout $(44,45)$. This observation can explain the reversion of the increased clearance seen on the day after exercise, and supports the concept of a post-exercise redistribution of TG FA. Moreover, LPL activity is reduced in skeletal muscle and up-regulated in adipose tissue when athletes detrain (46), again indicating a differentiation in lipid distribution (i.e. deposition vs oxidation) depending on the time elapsed since the last exercise bout.

There are limitations to our study. First, due to the small sample size a type 2 error cannot be excluded; yet tracer studies in combination with supervised long-term training are demanding and therefore limits the ability to recruit large numbers of participants. On the other hand, a sufficient number of subjects were included to detect significant differences in $\mathrm{VO}_{2} \mathrm{max}$ and insulin sensitivity. Second, there is a possibility of tracer recycling. However, in constant-infusion studies recycling of labeled VLDL-TG compose a minor part of steady state VLDL-TG SA, which is used for calculation of hepatic VLDL-TG secretion (47). Third, as mentioned, we cannot extend our findings to other forms of exercise, e.g. resistance exercise. Also, we do not have measurements of daily activity, and it is possible that our participants have reached a chronic high lowintensity exercise level with a near-maximum VLDL-TG metabolism. Therefore, we cannot extend the present findings to subjects with minimal daily activity. Fourth, it is possible that the result would have been different if the population investigated were either female, obese, had increased intrahepatic fat content, increased basal VLDL-TG secretion, or were insulin resistant. Fifth, no registration of food consumption was done in this study. However, both diet composition and calorie consumption can affect fasting TG synthesis. Consumption of a carbohydrate enriched diet results in a greater amount of glucose-derived FA TG without altering overall TG production, unless a hypercaloric diet is ingested (48). In addition, day-to-day variability of fasting VLDL-TG secretion rate in healthy volunteers (similar to those in our study), who were dietary controlled the day before the kinetic measurements, has previously been assessed to be around 15\% (49), i.e. similar to our findings. Although we did not record food consumption in this study, we believe that given the constant body weight before and after the intervention periods lends some confidence to a stable dietary intake during the study period.

In summary, 10 weeks of endurance training did not change VLDL-TG secretion, clearance and oxidation or palmitate turnover and oxidation rate despite improved insulin sensitivity and physical fitness. We conclude that the present persistent improvements in maximal oxygen uptake $(\approx 20 \%)$ and insulin sensitivity $(\approx 60 \%)$ do not induce persistent changes in VLDL-TG metabolism 60-72 h after the last exercise bout. Probably, endurance exercise must be performed frequently to maintain a favorable impact on VLDL-TG metabolism.

\section{Declaration of interest}

The authors declare that there is no conflict of interest that could be perceived as prejudicing the impartiality of the research reported.

\section{Funding}

The study has been financially supported by the World Anti-Doping Agency, The Novo Nordic Foundation, and The Danish Medical Research Council.

Author contribution statement

B Nellemann designed the study, conducted the study, researched the data and wrote the manuscript. B Christensen designed the study, conducted the study, contributed to the discussion, and reviewed the manuscript. $\mathrm{K}$ Vissing designed the exercise training protocol, contributed to the discussion, and reviewed the manuscript. L Thams, P Sieljacks, and M S Larsen conducted the study, and reviewed the manuscript. J O L Jørgensen contributed to the discussion, and reviewed the manuscript. $\mathrm{S}$ Nielsen researched the data, contributed to the discussion, and reviewed the manuscript.

\section{Acknowledgements}

The authors thank Lone Kvist, Susanne Sørensen, Elsebeth Hornemann, Hanne F Petersen, Kirsten Nyborg Rasmussen, and Eva Schriver for excellent technical assistance.

\section{References}

1 Jensen MD. Fate of fatty acids at rest and during exercise: regulatory mechanisms. Acta Physiologica Scandinavica 2003178 385-390. (doi:10.1046/j.1365-201X.2003.01167.x)

2 Kiens B. Effect of endurance training on fatty acid metabolism: local adaptations. Medicine and Science in Sports and Exercise 199729 640-645. (doi:10.1097/00005768-199705000-00009)

3 Austin MA, Hokanson JE \& Edwards KL. Hypertriglyceridemia as a cardiovascular risk factor. American Journal of Cardiology $1998 \mathbf{8 1}$ 7B-12B. (doi:10.1016/S0002-9149(98)00031-9)

4 Annuzzi G, Jansson E, Kaijser L, Holmquist L \& Carlson LA. Increased removal rate of exogenous triglycerides after prolonged exercise in man: time course and effect of exercise duration. Metabolism 198736 438-443. (doi:10.1016/0026-0495(87)90040-0)

5 Cullinane E, Siconolfi S, Saritelli A \& Thompson PD. Acute decrease in serum triglycerides with exercise: is there a threshold for an exercise effect? Metabolism 198231 844-847. (doi:10.1016/0026-0495(82) 90085-3) 
6 Tsetsonis NV \& Hardman AE. Effects of low and moderate intensity treadmill walking on postprandial lipaemia in healthy young adults. European Journal of Applied Physiology and Occupational Physiology 1996 73 419-426. (doi:10.1007/BF00334418)

7 Zhang JQ, Ji LL, Fogt DL \& Fretwell VS. Effect of exercise duration on postprandial hypertriglyceridemia in men with metabolic syndrome. Journal of Applied Physiology 2007103 1339-1345. (doi:10.1152/ japplphysiol.00181.2007)

8 Magkos F, Tsekouras YE, Prentzas KI, Basioukas KN, Matsama SG, Yanni AE, Kavouras SA \& Sidossis LS. Acute exercise-induced changes in basal VLDL-triglyceride kinetics leading to hypotriglyceridemia manifest more readily after resistance than endurance exercise. Journal of Applied Physiology 2008105 1228-1236. (doi:10.1152/japplphysiol. 90761.2008)

9 Magkos F, Wright DC, Patterson BW, Mohammed BS \& Mittendorfer B. Lipid metabolism response to a single, prolonged bout of endurance exercise in healthy young men. American Journal of Physiology. Endocrinology and Metabolism 2006290 E355-E362. (doi:10.1152/ ajpendo.00259.2005)

10 Morio B, Holmback U, Gore D \& Wolfe RR. Increased VLDL-TAG turnover during and after acute moderate-intensity exercise. Medicine and Science in Sports and Exercise 200436 801-806. (doi:10.1249/01.MSS. 0000126498.04454.C0)

11 Tsekouras YE, Magkos F, Prentzas KI, Basioukas KN, Matsama SG, Yanni AE, Kavouras SA \& Sidossis LS. A single bout of whole-body resistance exercise augments basal VLDL-triacylglycerol removal from plasma in healthy untrained men. Clinical Science 2009116 147-156. (doi:10.1042/CS20080078)

12 Al-Shayji IA, Caslake MJ \& Gill JM. Effects of moderate exercise on VLDL(1) and Intralipid kinetics in overweight/obese middle-aged men. American Journal of Physiology. Endocrinology and Metabolism 2012302 E349-E355. (doi:10.1152/ajpendo.00498.2011)

13 Barsalani R, Chapados NA \& Lavoie JM. Hepatic VLDL-TG production and MTP gene expression are decreased in ovariectomized rats: effects of exercise training. Hormone and Metabolic Research 201042 860-867. (doi:10.1055/s-0030-1267173)

14 Chapados NA, Seelaender M, Levy E \& Lavoie JM. Effects of exercise training on hepatic microsomal triglyceride transfer protein content in rats. Hormone and Metabolic Research 200941 287-293. (doi:10.1055/ s-0028-1102937)

15 Mondon CE, Dolkas CB, Tobey T \& Reaven GM. Causes of the triglyceride-lowering effect of exercise training in rats. Journal of Applied Physiology 198457 1466-1471.

16 Yoshida H, Ishikawa T, Suto M, Kurosawa H, Hirowatari Y, Ito K, Yanai H, Tada N \& Suzuki M. Effects of supervised aerobic exercise training on serum adiponectin and parameters of lipid and glucose metabolism in subjects with moderate dyslipidemia. Journal of Atherosclerosis and Thrombosis 201017 1160-1166. (doi:10.5551/jat.4358)

17 Durstine JL, Grandjean PW, Cox CA \& Thompson PD. Lipids, lipoproteins, and exercise. Journal of Cardiopulmonary Rehabilitation 200222 385-398. (doi:10.1097/00008483-200211000-00002)

18 Alam S, Stolinski M, Pentecost C, Boroujerdi MA, Jones RH, Sonksen PH $\&$ Umpleby AM. The effect of a six-month exercise program on very low-density lipoprotein apolipoprotein B secretion in type 2 diabetes. Journal of Clinical Endocrinology and Metabolism 200489 688-694. (doi:10.1210/jc.2003-031036)

19 Tsekouras YE, Magkos F, Kellas Y, Basioukas KN, Kavouras SA \& Sidossis LS. High-intensity interval aerobic training reduces hepatic very low-density lipoprotein-triglyceride secretion rate in men. American Journal of Physiology. Endocrinology and Metabolism 2008295 E851-E858. (doi:10.1152/ajpendo.90545.2008)

20 Sullivan S, Kirk EP, Mittendorfer B, Patterson BW \& Klein S.

Randomized trial of exercise effect on intrahepatic triglyceride content and lipid kinetics in nonalcoholic fatty liver disease. Hepatology 201255 1738-1745. (doi:10.1002/hep.25548)
21 Gormsen LC, Jensen MD \& Nielsen S. Measuring VLDL-triglyceride turnover in humans using ex vivo-prepared VLDL tracer. Journal of Lipid Research 200647 99-106. (doi:10.1194/jlr.M500205-JLR200)

22 Sondergaard E, Rahbek I, Sorensen LP, Christiansen JS, Gormsen LC, Jensen MD \& Nielsen S. Effects of exercise on VLDL-triglyceride oxidation and turnover. American Journal of Physiology. Endocrinology and Metabolism 2011300 E939-E944. (doi:10.1152/ajpendo.00031. 2011)

23 Sorensen LP, Andersen IR, Sondergaard E, Gormsen LC, Schmitz O, Christiansen JS \& Nielsen S. Basal and insulin mediated VLDLtriglyceride kinetics in type 2 diabetic men. Diabetes $20116088-96$. (doi:10.2337/db10-0564)

24 Gormsen LC, Nellemann B, Sorensen LP, Jensen MD, Christiansen JS \& Nielsen S. Impact of body composition on very-low-density lipoprotein-triglycerides kinetics. American Journal of Physiology. Endocrinology and Metabolism 2009296 E165-E173. (doi:10.1152/ajpendo.90675. 2008)

25 Mikines KJ, Sonne B, Farrell PA, Tronier B \& Galbo H. Effect of physical exercise on sensitivity and responsiveness to insulin in humans. American Journal of Physiology 1988254 E248-E259.

26 Perseghin G, Price TB, Petersen KF, Roden M, Cline GW, Gerow K, Rothman DL \& Shulman GI. Increased glucose transport-phosphorylation and muscle glycogen synthesis after exercise training in insulinresistant subjects. New England Journal of Medicine 1996335 1357-1362. (doi:10.1056/NEJM199610313351804)

27 Choi SH \& Ginsberg HN. Increased very low density lipoprotein (VLDL) secretion, hepatic steatosis, and insulin resistance. Trends in Endocrinology and Metabolism 201122 353-363. (doi:10.1016/j.tem. 2011.04.007)

28 Sorensen LP, Sondergaard E, Nellemann B, Christiansen JS, Gormsen LC \& Nielsen S. Increased VLDL-triglyceride secretion precedes impaired control of endogenous glucose production in obese, normoglycemic men. Diabetes 201160 2257-2264. (doi:10.2337/db110040)

29 Christensen B, Nellemann B, Larsen MS, Thams L, Sieljacks P, Vestergaard PF, Bibby BM, Vissing K, Stodkilde-Jorgensen H, Pedersen SB et al. Whole body metabolic effects of prolonged endurance training in combination with erythropoietin treatment in humans: a randomized placebo controlled study. American Journal of Physiology. Endocrinology and Metabolism 2013305 E879-E889. (doi:10.1152/ ajpendo.00269.2013)

30 Vissing K, McGee SL, Farup J, Kjolhede T, Vendelbo MH \& Jessen N. Differentiated mTOR but not AMPK signaling after strength vs endurance exercise in training-accustomed individuals. Scandinavian Journal of Medicine \& Science in Sports 201323 355-366.

31 Guo Z, Nielsen S, Burguera B \& Jensen MD. Free fatty acid turnover measured using ultralow doses of $\left[\mathrm{U}_{-}{ }^{13} \mathrm{C}\right]$ palmitate. Journal of Lipid Research 199738 1888-1895.

32 Guo Z, Burguera B \& Jensen MD. Kinetics of intramuscular triglyceride fatty acids in exercising humans. Journal of Applied Physiology 200089 2057-2064.

33 Ferrannini E. The theoretical bases of indirect calorimetry: a review. Metabolism 198837 287-301. (doi:10.1016/0026-0495(88)90110-2)

34 Sidossis LS, Coggan AR, Gastaldelli A \& Wolfe RR. A new correction factor for use in tracer estimations of plasma fatty acid oxidation. American Journal of Physiology 1995269 E649-E656.

35 Koutsari C, Ali AH, Mundi MS \& Jensen MD. Measuring plasma fatty acid oxidation with intravenous bolus injection of ${ }^{3} \mathrm{H}$ - and ${ }^{14} \mathrm{C}$-fatty acid. Journal of Lipid Research $2013 \mathbf{5 4} 254-264$. (doi:10.1194/jlr. P031153)

36 Gavin C, Sigal RJ, Cousins M, Menard ML, Atkinson M, Khandwala F, Kenny GP, Proctor S \& Ooi TC. Resistance exercise but not aerobic exercise lowers remnant-like lipoprotein particle cholesterol in type 2 diabetes: a randomized controlled trial. Atherosclerosis 2010213 552-557. (doi:10.1016/j.atherosclerosis.2010.08.071) 
37 Pitsavos C, Panagiotakos DB, Tambalis KD, Chrysohoou C, Sidossis LS, Skoumas J \& Stefanadis C. Resistance exercise plus to aerobic activities is associated with better lipids' profile among healthy individuals: the ATTICA study. QJM 2009102 609-616. (doi:10.1093/qjmed/hcp083)

38 Blaak EE, van Aggel-Leijssen DP, Wagenmakers AJ, Saris WH \& van Baak MA. Impaired oxidation of plasma-derived fatty acids in type 2 diabetic subjects during moderate-intensity exercise. Diabetes 200049 2102-2107. (doi:10.2337/diabetes.49.12.2102)

39 Horowitz JF \& Klein S. Lipid metabolism during endurance exercise. American Journal of Clinical Nutrition 200072 558S-563S.

40 Helge JW, Watt PW, Richter EA, Rennie MJ \& Kiens B. Fat utilization during exercise: adaptation to a fat-rich diet increases utilization of plasma fatty acids and very low density lipoprotein-triacylglycerol in humans. Journal of Physiology 2001537 1009-1020. (doi:10.1113/ jphysiol.2001.012933)

41 Roberts R, Bickerton AS, Fielding BA, Blaak EE, Wagenmakers AJ, Chong MF, Gilbert M, Karpe F \& Frayn KN. Reduced oxidation of dietary fat after a short term high-carbohydrate diet. American Journal of Clinical Nutrition 200887 824-831.

42 Krag MB, Gormsen LC, Guo Z, Christiansen JS, Jensen MD, Nielsen S \& Jorgensen JO. Growth hormone-induced insulin resistance is associated with increased intramyocellular triglyceride content but unaltered VLDL-triglyceride kinetics. American Journal of Physiology. Endocrinology and Metabolism 2007292 E920-E927. (doi:10.1152/ajpendo. 00374.2006)
43 Moller N \& Jorgensen JO. Effects of growth hormone on glucose, lipid, and protein metabolism in human subjects. Endocrine Reviews 200930 152-177. (doi:10.1210/er.2008-0027)

44 Kiens B \& Richter EA. Utilization of skeletal muscle triacylglycerol during postexercise recovery in humans. American Journal of Physiology 1998275 E332-E337.

45 Seip RL, Mair K, Cole TG \& Semenkovich CF. Induction of human skeletal muscle lipoprotein lipase gene expression by short-term exercise is transient. American Journal of Physiology 1997272 E255-E261.

46 Simsolo RB, Ong JM \& Kern PA. The regulation of adipose tissue and muscle lipoprotein lipase in runners by detraining. Journal of Clinical Investigation 199392 2124-2130. (doi:10.1172/JCI116813)

47 Sorensen LP, Gormsen LC \& Nielsen S. VLDL-TG kinetics: a dual isotope study for quantifying VLDL-TG pool size, production rates, and fractional oxidation in humans. American Journal of Physiology. Endocrinology and Metabolism 2009297 E1324-E1330. (doi:10.1152/ ajpendo.00366.2009)

48 Nielsen S \& Karpe F. Determinants of VLDL-triglycerides production. Current Opinion in Lipidology 201223 321-326. (doi:10.1097/MOL. Ob013e3283544956)

49 Magkos F, Patterson BW \& Mittendorfer B. Reproducibility of stable isotope-labeled tracer measures of VLDL-triglyceride and VLDLapolipoprotein B-100 kinetics. Journal of Lipid Research $2007 \mathbf{4 8}$ 1204-1211. (doi:10.1194/jlr.D600048-JLR200)

Received 24 April 2014

Revised version received 2 August 2014

Accepted 8 August 2014 